\title{
Femoral stem fractures after primary and revision hip replacements: A single-center experience
}

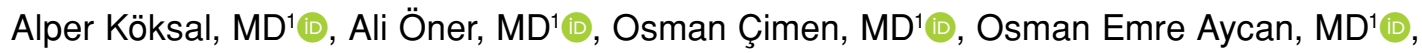 \\ Hakan Akgün, MD $^{1}{ }^{(1)}$, Furkan Yapıcı, MD $^{2}$ (D), Yalkın Çamurcu, MD $^{2}$ (D) \\ 1Department of Orthopedics and Traumatology, Istanbul Metin Sabancı Baltalimani Bone Diseases Training and \\ Research Hospital, Istanbul, Turkey \\ ${ }^{2}$ Department of Orthopedics and Traumatology, Erzincan Binali Yıldırım University Faculty of Medicine, Erzincan, Turkey
}

Femoral stem fracture is a devastating complication in hip replacement. Charnley ${ }^{[1]}$ reported $0.23 \%$ femoral stem fracture following hip replacement, while an another study indicated a ratio as high as $11 \% .{ }^{[2]}$ Femoral stem fractures used to be a common complication of first-generation forged stainless steel or casted cobalt chrome femoral stems. ${ }^{[3]}$ The development of new-generation femoral components in total hip arthroplasty has thus significantly reduced the rate of femoral stem fractures. ${ }^{[3]}$

As a result of the recent advances in design, metallurgy, and cementing techniques, femoral stem fractures following total hip arthroplasty have become rare. ${ }^{[4]}$ The American Association of Hip and Knee Surgeons reported $0.27 \%$ (172 in 64,483 cases) femoral stem fracture cases. ${ }^{[5]}$

Received: May 12, 2020

Accepted: June 20, 2020

Published online: September 11, 2020

Correspondence: Alper Köksal, MD. İstanbul Metin Sabancı Baltalimanı Kemik Hastalıkları Eğitim ve Araștırma Hastanesi Ortopedi ve Travmatoloji Kliniği, 34335 Beşiktaş, İstanbul, Türkiye.

E-mail: dralperkoksal@gmail.com

Doi: $10.5606 /$ ehc. 2020.76162

Citation: Köksal A, Öner A, Çimen O, Aycan OE, Akgün H, Yapıcı F, Çamurcu Y. Femoral stem fractures after primary and revision hip replacements: A single-center experience. Jt Dis Relat Surg 2020;31(3):557-563.

()2020 All right reserved by the Turkish Joint Diseases Foundation

This is an open access article under the terms of the Creative Commons Attribution-NonCommercial License, which permits use, distribution and reproduction in any medium, provided the original work is properly cited and is not used for commercial purposes (http://creativecommons.org/licenses/by-nc/4.0/)

\section{ABSTRACT}

Objectives: This study aims to present our experience in the management of fractured femoral stems after primary and revision hip replacements by evaluating the clinical and radiographic characteristics and determining the effectiveness of the extraction methods.

Patients and methods: A total of 15 patients (5 males, 10 females; mean age 65.9 years; range, 49 to 87 years) who underwent revision hip replacement due to a fractured femoral stem between January 2005 and December 2019 were included in this retrospective study. The mechanisms and risk factors for failure as well as methods applied to extract fractured stem were analyzed through clinical and radiographic data.

Results: Nine patients had fractured cemented femoral stems, while six patients had fractured fully porous coated cementless revision stems. Lack of proximal buttress in distally fixed femoral stems was detected in 11 patients and identified as the predominant mechanism resulting in fracture. The proximal extraction method with conventional revision instrumentation, the cortical window technique, and extended trochanteric osteotomy (ETO) were used in three, seven, and five cases, respectively.

Conclusion: Our results demonstrated that the lack of proximal buttress is the most common reason for femoral stem fracture. Moreover, the proximal extraction method was mostly ineffective in fully porous femoral stems. A step-by-step approach should be considered for the extraction of a broken stem. The cortical window method can be considered as the second step if proximal extraction methods fail, and ETO should be considered at the last step if all techniques fail.

Keywords: Femoral stem, stem fracture, stem revision, total hip arthroplasty.

The Swedish Hip Arthroplasty Register reported 140 revision surgeries due to stem fractures among 80,000 primary hip arthroplasty cases $(0.17 \%) .^{[6]}$ Stems generally fail due to a fatigue mechanism generated by unfavorable biomechanics, including varus positioning, loosening, loss of proximal 
buttress, unfavorable geometry of the stem, or surface damage of the implant. ${ }^{[7]}$ In some series, fracture initiation has been attributed to stress risers produced by laser etching, surface damage, defects in implant design, material microstructure, or machining. ${ }^{[8]}$

Lakstein et al. ${ }^{[9]}$ assessed six patients with femoral stem fracture, and the authors encountered early subsidence of femoral stem in one patient at postoperative fourth month after revision arthroplasty. Recently, Matar et al. ${ }^{[10]}$ reported that patients with either cemented or cementless stem fracture after primary arthroplasty had good midterm outcomes. However, the authors mentioned that patients with fractured femoral stem after revision arthroplasty had the poorest clinical outcome. ${ }^{[10]}$

According to our recent 10-year literature review, the clinical data about the management of fractured femoral components with the latest designs after primary and revision hip arthroplasties are limited..$^{[9-12]}$ Therefore, in this study, we aimed to present our experience in the management of fractured femoral stems after primary and revision hip replacements by evaluating the clinical and radiographic characteristics and determining the effectiveness of the extraction methods.

\section{PATIENTS AND METHODS}

In this retrospective study, the clinical data of patients who underwent revision hip arthroplasty between January 2005 and December 2019 were collected through the medical records of Health Sciences University, Baltalimani Bone and Joint Diseases Training and Research Hospital. A total of 722 revision hip arthroplasties were performed during this period. Fifteen patients (5 males, 10 females; mean age 65.9 years; range, 49 to 87 years) who underwent revision hip arthroplasty due to fractured femoral components after primary or revision total hip arthroplasty were included in the study. The study protocol was approved by the Health Sciences University, Baltalimani Bone and Joint Diseases Training and Research Hospital Ethics Committee (Approval no: 316). Due to the retrospective design of the study, there was no requirement for the patient consent. The study was conducted in accordance with the principles of the Decleration of Helsinki.

Patients' demographics, clinical characteristics, operative data, and postoperative follow-up medical records were reviewed. The implants used in the primary surgery were also recorded. Detailed radiographic examination was performed through preoperative radiographs. Femoral bone defects were classified according to the Paprosky classification of femoral bone loss, which was described by Aribindi et al. ${ }^{[13]}$ and categorized into four types (Table I). Four senior surgeons in the field of arthroplasty reviewed the preoperative radiographs and clinical data to reach a consensus about the failure mechanism. Failure mechanism was proved if three or more surgeons agreed about the diagnosis. No inter-observer or intra-observer correlation analysis was performed between observers.

All patients received prophylactic firstgeneration cephalosporin 30 minutes prior to their procedures. Five patients were operated on the left hip, and 10 patients were operated on the right hip under general anesthesia. All surgeries were performed through a standard posterolateral approach. A total of nine patients had fractured cemented femoral stems, while six patients had fractured fully porous coated cementless revision stems, which were obtained from different manufacturers for their previous operations (Figures 1 and 2). In nine patients with fractured cemented femoral stems, the localizations of the femoral component fractures were as follows: at the proximal third in two patients, at the middle third in three patients, at the distal third in two patients, and at the stem-neck junction in two patients. Fractured femoral components were extracted by the cortical window method in four patients, by the extended trochanteric osteotomy (ETO) method in two patients, and by proximal extraction in three patients. In six patients with fractured fully porous coated cementless revision stems, the stem fractures were localized at the proximal third in four patients and at the middle third in two patients. The fractured stems were extracted by

\section{TABLE I}

Paprosky classification of femoral bone loss ${ }^{[13]}$

Type I Minimal metaphyseal bone loss, diaphysis is intact

Type II Metaphyseal bone loss with minimal diaphyseal damage

Type IIla Metaphyseal and diaphyseal bone loss, $\geq 4 \mathrm{~cm}$ intact diaphyseal bone near isthmus

Type IIlb Metaphyseal and diaphyseal bone loss, $<4 \mathrm{~cm}$ intact diaphyseal bone near isthmus

Type IV Extensive metaphyseal and diaphyseal bone loss, damaged isthmus 

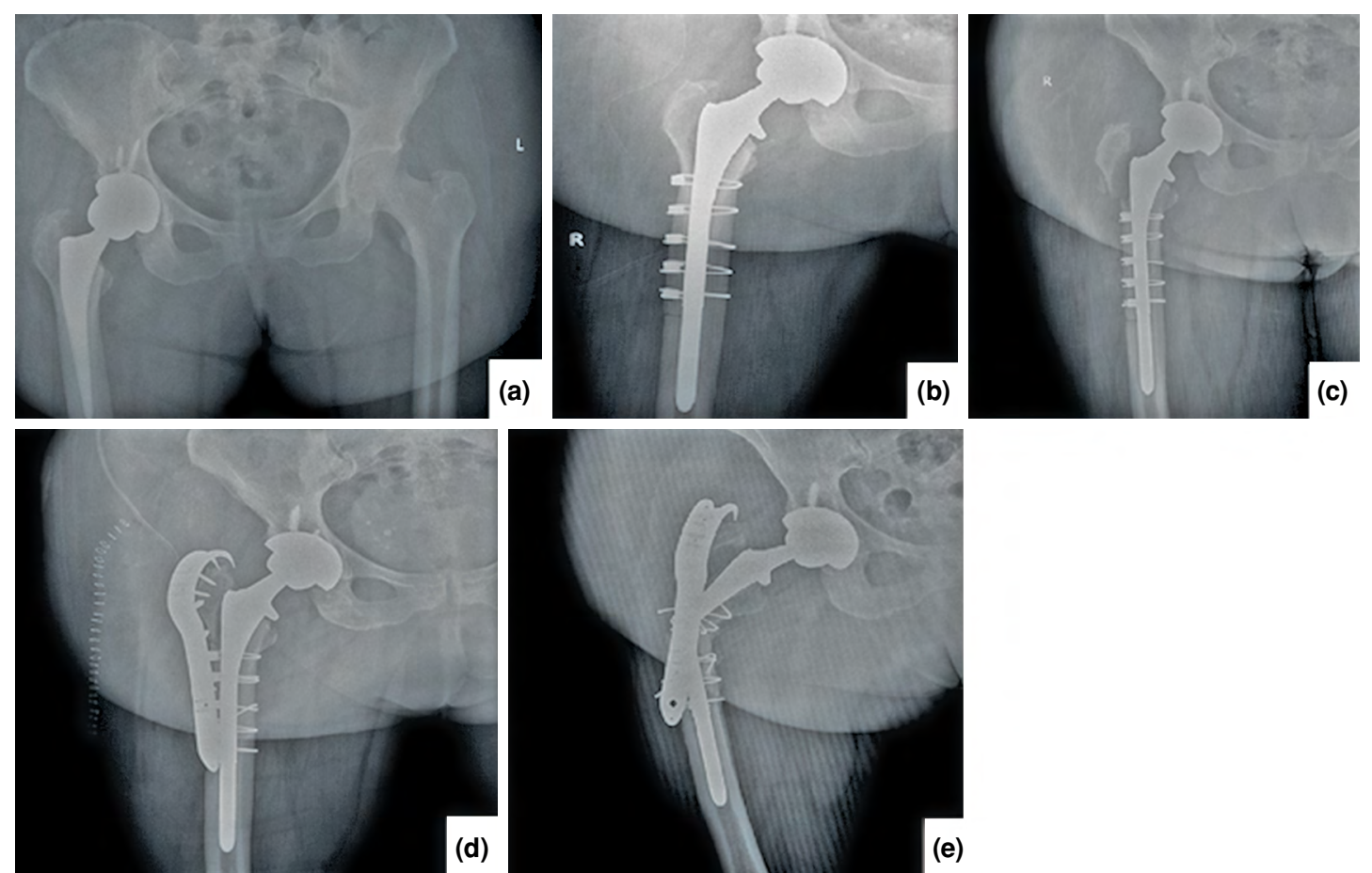

FIGURE 1. (a, b) A 62-year-old female patient who underwent revision hip arthroplasty with a diagnosis of aseptic loosening of acetabulum in 2017. (c, d) In 2018, a trochanter major fracture occurred, and a trochanteric plate was applied. (e) One year after trochanter major fixation in 2019, patient had a femoral stem fracture due to proximal support deficiency.

the cortical window method in three cases and by the ETO method in three cases. A fully porous monoblock revision femoral stem was used for all patients in the revision arthroplasty procedure. Fixation of the bone block after ETO was performed by cable fixation in all three patients. Moreover, a cable-plate fixation was performed in two patients and a cable fixation was performed in four patients, due to severe bone loss to secure periprosthetic fracture.

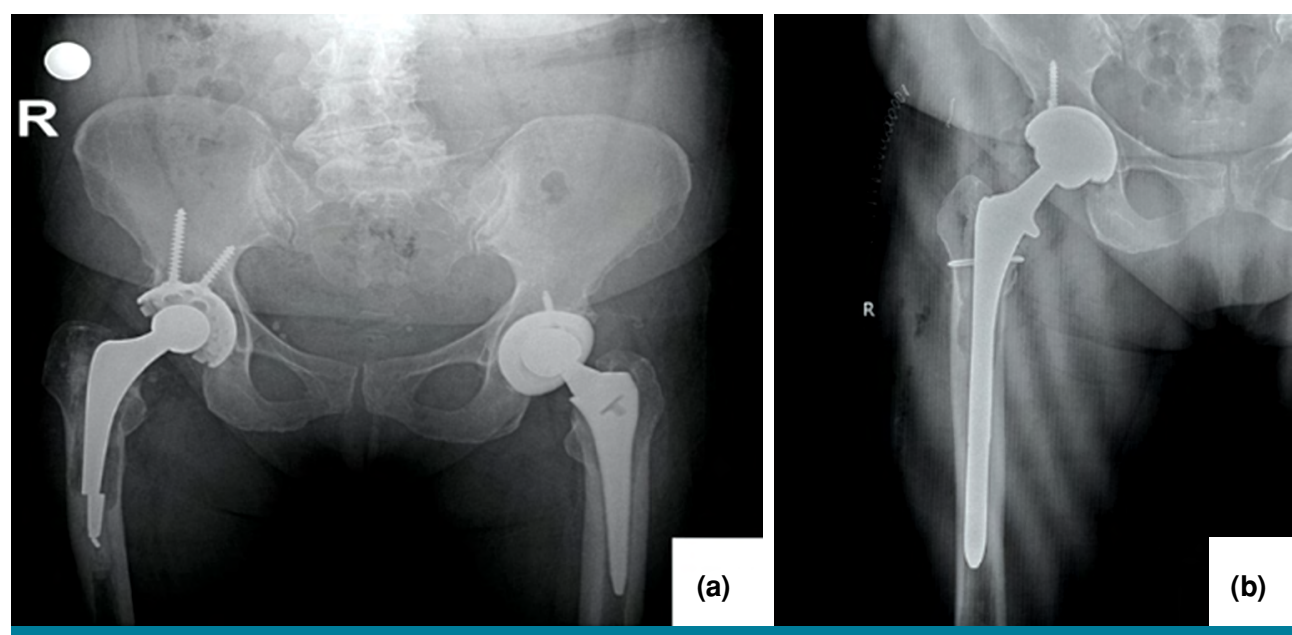

FIGURE 2. (a) A 59-year-old female patient who underwent primary hip arthroplasty with a cemented femoral stem in 2001. In 2015, patient had aseptic loosening and a femoral stem fracture at distal third of stem due to proximal support deficiency. (b) Broken stem was extracted with proximal distraction method without need for corticotomy, and a revision prosthesis was applied. 
Postoperative intravenous first-generation cephalosporin was given for 24 hours. All patients received low-molecular-weight heparin for thromboembolic prophylaxis until the end of the fourth postoperative week. Hip range of motion exercises were started on the second postoperative day, and weight bearing was allowed at the sixth postoperative week following the ETO or cortical window procedure. The first follow-up after surgery was performed on the $15^{\text {th }}$ postoperative day. Subsequent follow-up visits were planned in the first, third, and sixth postoperative months and annually thereafter. Clinical and radiographic examinations were performed and recorded at each visit. We did not use any clinical scores to assess the functional outcomes; we rather preferred to record patients' self-reported complaints. Patients' latest follow-up radiographs were assessed, and acetabular and femoral osteolysis and loosening were noted if they existed. All medical complications such as deep venous thrombosis and pulmonary embolus as well as prosthesis related complications such as deep infection, dislocation, and implant failures were also recorded.

\section{RESULTS}

The clinical characteristics of the patients are presented in Table II. The fractures of the femoral components were located at the femoral neck-stem junction in two patients, at the proximal third of the femoral stem in six patients, at the middle third in five patients, and at the distal third in two patients. Femoral bone defects were classified as Paprosky type I in eight patients, type II in five patients, and type IIIA in two patients.

The lack of proximal bone stock in distally fixed stems was detected in 11 patients, and it was identified as the predominant mechanism for the femoral stem fracture. Fracture due to varus malposition of the femoral stem was detected in two patients. Two patients had no other pathology explaining the occurrence of stem fracture. Both patients had appropriate-sized stems, good cementing techniques, correct placement of the femoral components at an appropriate angle, and did not have any signs of loosening. These patients with fractures at the femoral neck-stem junctions were obese with body mass indexes (BMIs) over $40 \mathrm{~kg} / \mathrm{m}^{2}$.

Two patients had dislocations of the femoral head on the fifth and $14^{\text {th }}$ postoperative days, and both underwent closed reduction under general anesthesia. An anti-rotation cast was applied, and these patients were immobilized in bed rest for three weeks. No re-dislocation was observed during their follow-ups. One patient underwent debridement due to prolonged drainage from the wound on the seventh postoperative day and successfully recovered without the need for a secondary intervention (microbiologic culture negative). Prolonged drainage was defined as wound drainage until postoperative fifth day, and inflammatory markers were not considered in decision making due to postoperative normal increment. One patient who had femoral stem fracture after two-stage revision of periprosthetic infection had periprosthetic infection again. This patient underwent removal of the prosthesis and application of a handmade antibiotic-loaded cement spacer. He refused to undergo revision again due to having no complaint and pain despite full weight bearing. No systemic medical complications occurred during follow-up. At the latest follow-up, which ranged from one year to 10 years, all patients were actively mobile without the need for any device and they had no prosthesis related complaint. Moreover, no severe osteolysis or loosening which needed revision surgery was examined at the last follow-up radiographs.

\section{DISCUSSION}

The most important finding of this study was observing femoral stem fractures mostly in patients with the lack of proximal buttress. ${ }^{[14]}$ Moreover, the proximal distraction method was mostly ineffective and had been performed in three patients with broken cemented femoral stems. Large nationwide studies reported that femoral stem fractures vary from 0.17 to $0.27 \%{ }^{[5,6]}$ The low rate of this devastating complication can be explained by recent stem designs and meticulous surgical techniques. However, our results revealed a $2.07 \%$ rate of revision hip arthroplasty between 2005 and 2019 due to femoral stem fracture; such rate is higher than that of the National Registries. This ratio may be attributed to the referral of patients from other hospitals because our institution is a tertiary referral center for joint replacement surgery.

The etiology of femoral stem fractures is thought to be multifactorial. ${ }^{[15]}$ Yates et al..$^{[8]}$ reported that an inadequate cement mantle in the proximal medial femur entails a risk for critical overload of the distal cement, mantle fracture, loss of proximal support, cantilever bending, and, consequently, fracture of the stem. Jazrawi et al. ${ }^{[15]}$ claimed that the main mechanism of fracture is the use of small femoral stems loaded with excessive stress from patients' weights and activities. Wroblewski ${ }^{[16]}$ reported the largest series of fractured stems with 120 Charnley 


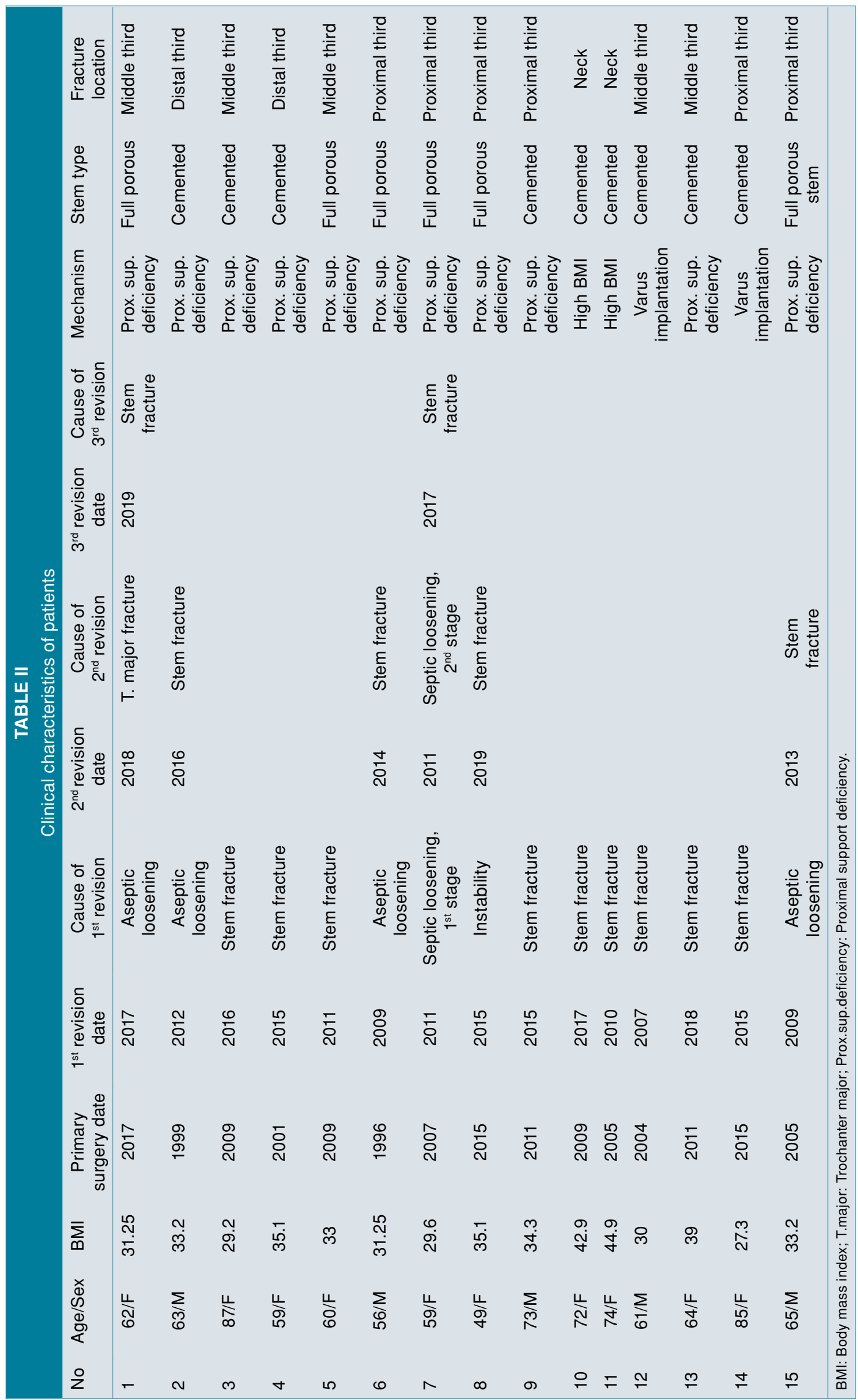


flat backs, which had a fracture rate of $1.15 \%$, and found that $77.2 \%$ of all stem fractures in the study had a failure of the femoral stem fixation within the first year of operation. The author also stated that valgus-positioned stems and overweight patients fractured relatively early, although the varus position was common. Wroblewski ${ }^{[16]}$ suggested that a patient's age and function are important factors. Poor surgical technique (femoral stem placed in the varus and inadequate cementing technique) is another risk factor for stem fracture. ${ }^{[8,14,17-19]}$ Ayers and Mann ${ }^{[20]}$ reported that varus mispositioning increases the tensile forces on an implant, increases the bending moment on the stem, and reduces the cement mantle in zone 7 around the calcar (particularly in the isthmus), thereby making the stem highly susceptible to fractures. Busch et al. ${ }^{[21]}$ found that fractured stems are associated with poor proximal buttress, BMI $>30$, small stem diameter $(<13.5 \mathrm{~mm})$, and increased need for the ETO method. Lakstein et al. ${ }^{[9]}$ identified excessive body weight, inadequate proximal buttress due to trochanteric osteotomy, reduced preoperative bone stock, osteolysis, loosening, and implant undersizing as risk factors for stem fracture. Matar et al. ${ }^{[10]}$ reported that the predominant mode of failure is the lack of proximal buttress in distally fixed stems and that most fractures occur in mid-stems. Buttaro et al. ${ }^{[12]}$ also remarked the absence of proximal femoral bone stock in the mechanism of failure. In our study, we also found that the predominant mode of failure is the lack of proximal buttress for both primary and revision hip arthroplasties. We observed femoral stem-neck fracture at two patients with BMIs $>40 \mathrm{~kg} / \mathrm{m}^{2}$. Bolland et al. ${ }^{[1]]}$ also mentioned that they observed neck fractures in $43 \%$ of their patients with increased BMI and/or activity level.

Wroblewski ${ }^{[22]}$ defined an extraction method that involves drilling holes at the fractured stem. Harris et al. $^{[23]}$ proposed the use of a wedged device to extract fractured stems. These drilling techniques are difficult to apply because recent stems are made of durable materials. Collis and Dubrul ${ }^{[24]}$ described the use of trephines to extract fractured stems. The cortical window method that is still being used today was proposed by Moreland et al. ${ }^{[25]}$ In 1995, Younger et al. ${ }^{[26]}$ described the ETO method for extracting fractured stems. In our series, we followed specific steps to remove fractured stems. First, we applied the proximal extraction technique with conventional revision instrumentations. In three cases (cemented stems), the fractured stem was extracted by this method; however, this technique was ineffective in other cases with fully porous stems. Second, we performed the extraction with the cortical window method. The cortical window was created with drill holes and saws to evaluate the distal end of the fractured stem. The stem was then removed by hitting toward the proximal from this window. In seven cases, the fractured stems were extracted by the cortical window method. As the application and subsequent femoral reconstruction are easier with the cortical window method than with the ETO technique, we preferred the former. In five cases, the cortical window method failed, and we performed ETO, which allows the direct visualization and removal of the distal fractured stem. Matar et al. ${ }^{[10]}$ performed proximal extraction method in five of 10 historical stems, five of 15 cemented stems, and five of 10 revision stems. The authors suggested proximal extraction method particularly in cases with previous trochanteric osteotomy, and preferred ETO in the last step. ${ }^{[10]}$

The main limitation of this study is its use of a small patient population relative to that in the National Registries. Nevertheless, we reported a single-center experience for the treatment of an unusual complication of total hip arthroplasty, which was treated by the same protocol. The heterogeneity of the implant designs used in the primary or revision hip arthroplasties is another limitation of the study. Nevertheless, our results yielded compatible information with the literature in terms of risk factors and offer a reliable reference for step-by-step femoral stem extraction during the challenging revision procedure. A meticulous cementation method, appropriate selection of femoral stem size, and neutral placement of the stem are the precautions that should be taken during primary hip replacement to reduce stem fractures. Good stiffness of the graft for proximal buttress and proper femoral reconstruction reduce the risk for femoral stem fractures in revision hip replacement. Reducing a patient's weight to an appropriate level and with an appropriate activity program may also help reduce the risk of femoral neck-stem junction fractures. Great care should be taken in considering revision hip replacement for the lack of proximal buttress in distally fixed stems, and the procedure should be planned accordingly. A step-by-step approach should be followed for the removal of fractured femoral stems. The proximal extraction method with conventional revision instrumentations should be the first line of approach, followed by the cortical window method. The ETO method may only be performed if all the other techniques fail. 
In conclusion, our results demonstrated that the lack of proximal buttress is the most common reason for femoral stem fracture. Moreover, the proximal extraction method was mostly ineffective in fully porous femoral stems. A step-by-step approach should be considered for the extraction of a broken stem. The cortical window method can be considered as the second step if proximal extraction methods fail, and ETO should be considered at the last step if all techniques fail.

\section{Declaration of conflicting interests}

The authors declared no conflicts of interest with respect to the authorship and/or publication of this article.

\section{Funding}

The authors received no financial support for the research and/or authorship of this article.

\section{REFERENCES}

1. Charnley J. Fracture of femoral prostheses in total hip replacement. A clinical study. Clin Orthop Relat Res 1975;111:105-20.

2. Martens M, Aernoudt E, de Meester P, Ducheyne P, Mulier JC, de Langh R, et al. Factors in the mechanical failure of the femoral component in total hip prosthesis. Report of six fatigue fractures of the femoral stem and results of experimental loading tests. Acta Orthop Scand 1974;45:693-710.

3. Dall DM, Learmonth ID, Solomon MI, Miles AW, Davenport JM. Fracture and loosening of Charnley femoral stems. Comparison between first-generation and subsequent designs. J Bone Joint Surg [Br] 1993;75:259-65.

4. Della Valle AG, Becksaç B, Anderson J, Wright T, Nestor B, Pellicci PM, et al. Late fatigue fracture of a modern cemented [corrected] cobalt chrome stem for total hip arthroplasty: a report of 10 cases. J Arthroplasty 2005;20:1084-8.

5. Heck DA, Partridge CM, Reuben JD, Lanzer WL, Lewis CG, Keating EM. Prosthetic component failures in hip arthroplasty surgery. J Arthroplasty 1995;10:575-80.

6. Swedish Hip Arthroplasty Register. Annual Report 2017, Available at: https://registercentrum.blob.core.windows. net/shpr/r/Eng_Arsrapport_2017_Hoftprotes_finalSyx2fJPhMN.pdf. [Accessed: January 28, 2019].

7. Wroblewski BM. The mechanism of fracture of the femoral prosthesis in total hip replacement. Int Orthop 1979;3:137-9.

8. Yates PJ, Quraishi NA, Kop A, Howie DW, Marx C, Swarts E. Fractures of modern high nitrogen stainless steel cemented stems: cause, mechanism, and avoidance in 14 cases. J Arthroplasty 2008;23:188-96.

9. Lakstein D, Eliaz N, Levi O, Backstein D, Kosashvili Y, Safir O, et al. Fracture of cementless femoral stems at the mid-stem junction in modular revision hip arthroplasty systems. J Bone Joint Surg [Am] 2011;93:57-65.
10. Matar HE, Selvaratnam V, Board TN, Purbach B, Porter ML, Kay PR, et al. Fractured Femoral Stems in Primary and Revision Hip Arthroplasties Revisited: Wrightington Experience. J Arthroplasty 2020;35:1344-50.

11. Bolland BJ, Wilson MJ, Howell JR, Hubble MJ, Timperley AJ, Gie GA. An Analysis of Reported Cases of Fracture of the Universal Exeter Femoral Stem Prosthesis. J Arthroplasty 2017;32:1318-22.

12. Buttaro M, Comba F, Zanotti G, Piccaluga F. Fracture of the C-Stem cemented femoral component in revision hip surgery using bone impaction grafting technique: report of 9 cases. Hip Int 2015;25:184-7.

13. Aribindi R, Barba M, Solomon MI, Arp P, Paprosky W. Bypass fixation. Orthop Clin North Am 1998;29:319-29.

14. Bozkurt HH, Tokgöz MA, Yapar A, Atik OŞ. What is the importance of canal-to-diaphysis ratio on osteoporosisrelated hip fractures? Eklem Hastalik Cerrahisi 2019;30:296-300.

15. Jazrawi LM, Della Valle CJ, Kummer FJ, Adler EM, Di Cesare PE. Catastrophic failure of a cemented, collarless, polished, tapered cobalt-chromium femoral stem used with impaction bone-grafting. A report of two cases. J Bone Joint Surg [Am] 1999;81:844-7.

16. Wroblewski BM. Fractured stem in total hip replacement. A clinical review of 120 cases. Acta Orthop Scand 1982;53:279-84.

17. Vatani N, Comando D, Acuña J, Prieto D, Caviglia H. Faulty design increases the risk of neck fracture in a hip prosthesis. Acta Orthop Scand 2002;73:513-7.

18. Carlsson AS, Gentz CF, Stenport J. Fracture of the femoral prosthesis in total hip replacement according to Charnley. Acta Orthop Scand 1977;48:650-5.

19. Galante JO. Causes of fractures of the femoral component in total hip replacement. J Bone Joint Surg [Am] 1980;62:670-3.

20. Ayers D, Mann K. The importance of proximal cement filling of the calcar region: a biomechanical justification. J Arthroplasty 2003;18(7 Suppl 1):103-9.

21. Busch CA, Charles MN, Haydon CM, Bourne RB, Rorabeck $\mathrm{CH}$, Macdonald SJ, et al. Fractures of distally-fixed femoral stems after revision arthroplasty. J Bone Joint Surg $[\mathrm{Br}]$ 2005;87:1333-6.

22. Wroblewski BM. A method of management of the fractured stem in total hip replacement. Clin Orthop Relat Res 1979;(141):71-3.

23. Harris WH, White RE Jr, Mitchel S, Barber F. A new technique for removal of broken femoral stems in total hip replacement. A technical note. J Bone Joint Surg [Am] 1981;63:843-5.

24. Collis D, Dubrul W. The removal of fractured prosthetic components from medullary cavities: a new technique. Comtemp Orthop 1984;8:61-5.

25. Moreland JR, Marder R, Anspach WE Jr. The window technique for the removal of broken femoral stems in total hip replacement. Clin Orthop Relat Res 1986;212:245-9.

26. Younger TI, Bradford MS, Magnus RE, Paprosky WG. Extended proximal femoral osteotomy. A new technique for femoral revision arthroplasty. J Arthroplasty 1995;10:329-38. 